\title{
Failed Etonogestrel Implant (Nexplanon) in a Woman on anti-TB Medications: A Case Report
}

Tesfaye H. Tufa ( $\nabla$ tesfayehurisa50@gmail.com )

Saint Paul's Hospital Millennium Medical College https://orcid.org/0000-0002-8623-9113

Abraham Fessehaye

St Paul's Hospital Millennium Medical College

Ferid A. Abubeker

St Paul's Hospital Millennium Medical College

\section{Case Report}

Keywords: Etonogestrel implant, Contraceptive failure, Anti-tuberculosis, Unintended pregnancy, Case report

Posted Date: January 18th, 2021

DOI: https://doi.org/10.21203/rs.3.rs-146071/v1

License: (c) (i) This work is licensed under a Creative Commons Attribution 4.0 International License. Read Full License 


\section{Abstract}

\section{Background}

A new form of implantable subdermal contraceptive device (Nexplanon) is available in the market since 2001 and provides $99 \%$ effective contraception. However, similar to other hormonal contraception, its effectiveness is compromised with the use of cytochrome P450 inducing drugs resulting in an unplanned pregnancy. Despite this theoretical risk, little is known on the outcome of concomitant use of antituberculosis medications and Implants.

\section{Case presentation}

A 26 years old GIII PII who has been using Nexplanon for the past 2 years and 7 months presented to the family planning referral clinic with the diagnosis of failed implant and second-trimester pregnancy at gestational age of 19 weeks. She was initiated on rifampicin based anti-tuberculosis 8 months prior to her presentation for the diagnosis of tuberculosis of the lymph nodes.

\section{Conclusion}

All health professionals should be aware of the possible reduced effectiveness of certain hormonal contraceptives due to concomitant use of hepatic cytochrome P450 enzyme-inducing medications. Women should be given detailed counseling about the potential for drug interaction. The discussion should include the use of other alternative methods. A multidisciplinary approach with consultation or referral to reproductive health specialists is crucial for optimal management of women who are at increased risk of contraceptive failure and unintended pregnancy.

\section{Introduction}

Long acting reversible contraceptives (LARCs) including Implants are among the most effective methods of reversible contraception with more than $99 \%$ efficacy [1]. It is a $4 \mathrm{~cm}$ long and $2 \mathrm{~mm}$ wide rod implant containing $68 \mathrm{mg}$ of etonogestrel and inserted in the inner aspect of the non-dominant hand [2]. The mechanism of action is mainly through suppression of ovulation augmented by increased cervical mucus viscosity that hinders the passage of spermatozoa and effectively prevents pregnancy up to four years $[2,3]$.

Cytochrome P 450 enzyme inducing medications including anti-TB are reported to be one of the reasons for failure of this highly effective method. These drugs reduce contraception effectiveness through activation of cytochrome $\mathrm{P} 450$ which in turn increases serum clearance of hormonal contraception making the hormones less available in the serum to avoid pregnancy [4].

We present a case of failed implant that highlights the importance of counseling by providers on possible drug interaction of contraception with concomitant use of implants and anti-tuberculosis drugs. 


\section{Case Presentation}

A 26 years old GIII PII woman was referred to Saint Paul's Hospital Millennium Medical College (SPHMMC) with a diagnosis of second-trimester pregnancy and failed Nexplanon.

Two and a half years back, she had uncomplicated vaginal delivery at SPHMMC. Following her delivery, she was counseled on possible options of contraception and opted for Nexplanon as she wanted to delay pregnancy for a minimum of three years. At the time of insertion of the Nexplanon, she had no medical illness or any contraindication for its use. The Nexplanon was well tolerated except reduction in the frequency and amount of menstrual bleeding, especially after the first year of use.

Eight months back, she was diagnosed with tuberculosis lymphadenitis and was initiated on rifampicin based anti-tuberculosis medications. During the initiation of anti-TB treatment, she claims that she was reassured about the effectiveness of Nexplanon and started the treatment without being informed about the possibility of unintended pregnancy. She was not informed to change the contraception method or use an additional method of contraception.

\section{Discussion}

Etonogestrel implant is one of the most effective long-acting reversible contraceptive methods. In most healthy women it is more than $99 \%$ effective with a failure rate lower than permanent sterilization techniques $[5,6]$.

Several factors have been implicated for the failure of implant contraceptives. In a review of more than 200 unintended pregnancies among women using etonogestrel implant, improper insertion was the most common reason for method failure accounting for $38 \%$ of cases whereas $4 \%$ of cases were due to drug interactions [7]. Another large study however showed that a large proportion of contraceptive failure (nearly $25 \%$ ) were associated with intake of drugs that can potentially affect contraceptive efficacy [8].

Etonogestrel is metabolized by the hepatic cytochrome P 450 (CYP450) enzyme system. Drugs that induce CYP 450 may increase the rate of hepatic degradation, leading to a lower bioavailability and potential loss of contraceptive effect [9]. For example, a pharmacologic study of women using etonogestrel implant showed a drop in the serum etonogestrel level below the threshold needed to inhibit ovulation after administration of carbamazepine, which is known to induce CYP450 enzyme system [10]. Similar findings of drug interaction and failed implants were reported among women on other antiepileptic, antiretroviral, and antituberculosis drugs [2,11,12]. Our patient was started on an antituberculosis regimen containing rifampicin, a known CYP450 enzyme inducer [9].

For women who are using or planning to use hormonal contraceptives, several considerations need to be made should they require treatment with medications that are CYP450 enzyme inducer. A multidisciplinary approach with consultation or referral to reproductive health specialists can play a significant role for optimal management. They need to receive detailed counseling about the potential for 
drug interaction. The discussion should include the use of other alternative methods. Both the WHO and US medical eligibility criteria (MEC) recommend women on implants who need long-term treatment with rifampicin to use other contraceptive methods $[13,14]$. The UK MEC advises the use of additional barrier methods or a switch to other methods when the potential for drug interactions is a concern $[15,16]$.

\section{Conclusion}

This case report highlights the need for detailed contraceptive counseling for women with medical comorbidities. Health care providers should be aware of the possible reduced effectiveness of certain hormonal contraceptives due to concomitant use of hepatic cytochrome P450 enzyme-inducing medications. Alternative contraceptive options should be offered in these cases. Thorough medical and contraceptive history with consultation or referral to reproductive health specialists is crucial for optimal management of women who are at increased risk of contraceptive failure and unintended pregnancy.

\section{Declarations}

\section{Ethics approval and consent to participate}

Ethical clearance was obtained from the Institutional Research and Ethics Review Committee (IRB) of SPHMMC for publication of the case report.

\section{Consent for publication}

Informed and written consent was taken from the patient to publish the case report

\section{Availability of data materials}

All datas materials related to the case report are included in the manuscript

\section{Conflict of interest}

All authors declare no conflict of interest.

\section{Funding}

The research did not receive a grant or any form of support from funding agents or authorities.

\section{Authors contribution}

THT was involved in patient care, conceived the study, did literature search, and drafted the manuscript. AF drafted the manuscript and critical revision of the manuscript. FAA drafted the manuscript, critical revision of the manuscript, and overall supervision of the manuscript. All authors have read and approved the manuscript 
Acknowledgments

We want to thank our patient for her consent in allowing us to share her experience as well as all health care providers who are involved in her care. We also thank the department of obstetrics and gynecology of Saint Paul's Hospital Millnnium Medical College.

\section{References}

[1] Croxatto HB. Mechanisms that explain the contraceptive action of progestin implants for women. Contraception. 2002;65:21-27.

[2] Gbolade B. Failure of Implanon \& reg; on antituberculous therapy. Open Access J Contracept 2010:103.

[3] Croxatto HB. Mechanisms that explain the contraceptive action of progestin implants for women. Contraception 2002;65:21-7. https://doi.org/10.1016/s0010-7824(01)00294-3.

[4] Zhang N, Shon J, Kim M, Yu C, Zhang L, Huang S, et al. Role of CYP3A in Oral Contraceptives Clearance. Clin Transl Sci 2018;11:251-60.

[6] Trussell J and Aiken ARA, Contraceptive efficacy. In: Hatcher RA et al. Contraceptive Technology, 21 st revised edition. New York, Ardent Media, 2018

[7] Harrison-Woolrych M, Hill R. Unintended pregnancies with the etonogestrel implant (Implanon): a case series from postmarketing experience in Australia. Contraception 2005;71:306-8. https://doi.org/10.1016/j.contraception.2004.10.005.

[9] Perry R, Stone RH, Haider S. Medication interactions with contraceptives. In: Allen RH, Cwiak CA, editors. Contraception for the medically challenging patient. New York: Springer Science + Business Media; 2014. p. 347-62..

[10] Lazorwitz A, Davis A, Swartz M, Guiahi M. The effect of carbamazepine on etonogestrel concentrations in contraceptive implant users. Contraception 2017;95:571-7. https://doi.org/10.1016/j.contraception.2017.03.004.

[11] Contraceptive implant failures among women... | Gates Open Research n.d. https://gatesopenresearch.org/articles/3-1482/v2 (accessed December 22, 2020).

[12] Lange J, Teal S, Tocce K. Decreased efficacy of an etonogestrel implant in a woman on antiepileptic medications: a case report. J Med Case Reports 2014;8:43. https://doi.org/10.1186/1752-1947-8-43.

[13] World Health Organization. 2015 Medical Eligibility Criteria for Contraceptive Use. 5th Edition. Geneva: World Health Organization; 2015. Available from: https://www.who.int/publications/i/item/9789241549158 (accessed on 24 December 2020). 
[14] Centers for Disease Control and Prevention. US Medical Eligibility Criteria (US MEC) for contraceptive use. USA: Division of Reproductive Health, National Center for Chronic Disease Prevention and Health Promotion, 2016.

[15] UKMEC April 2016 (Amended September 2019) - Faculty of Sexual and Reproductive Healthcare n.d. https://www.fsrh.org/standards-and-guidance/documents/ukmec-2016/ (accessed January 2, 2021).

[16] Drug Interactions - Faculty of Sexual and Reproductive Healthcare n.d. https://www.fsrh.org/standards-and-guidance/fsrh-guidelines-and-statements/drug-interactions/ (accessed January 2, 2021). 